\title{
AUTOMATED MODULATION CLASSIFICATION SYSTEM FOR SOFTWARE DEFINED RADIO
}

\author{
Dr. Hasin Alam, \\ Department of Electronics and Communication Engineering, \\ Ibra College of Technology, \\ Oman \\ alamhasin@ict.edu.om
}

\begin{abstract}
An Automatic Modulation Classification (AMC) system for Software Defined Radio (SDR) is presented in this study. Initially, the generated signals are modulated using different modulation techniques. Then, noise is added to the generated signals by using Additive White Gaussian Noise (AWGN). The noise added signal is used for further process to extract features and classification. The system uses Discrete Wavelet Transform (DWT) to analyze the signal that produces lower and higher frequency sub-bands. The Independent Component Analysis (ICA) is employed on lower frequency subband for dimensionality reduction. Finally, the classification is made by Pulse Coupled Neural Network (PCNN). The system uses three different digital modulation schemes; Phase Shift Keying (PSK), Quadrature Amplitude Modulation (QAM), and Differential PSK (DPSK). The results show the DWT, ICA and PCNN based AMC system provides promising results under various noise densities.
\end{abstract}

Keywords: Automated modulation classification, machine learning algorithms, dimensionality reduction techniques, software defined radio.

\section{INTRODUCTION}

The classification of digital signals using compressed cyclo-stationary features is described in [1]. The input signals are extracted by using time averaged cyclic periodogram. Then, DWT is used for decomposition. Neural network classifier is used for classification. Cognitive radio based automatic blind modulation recognition is described in [2]. The input signals are extracted by higher order cumulants, temporal time domain features. Decision tree algorithm is used for the classification of modulated signals.

Linearly modulated signals are described in a cognitive radio network in [3] The clustering based baseband symbols are used to recognize the signals. The $k$ means clustering and fuzzy c-means clustering method are used for validation. Automatic classification of modulated signals in cognitive radios by using the combination of blind equalization is described in [4]. The predictor-based recursive blind equalizer is used for feature extraction. The cyclo-stationary method is used for feature detection. The classification is made by probability of classification method.

DWT based modulated signal classification is described in [5]. The input signals are decomposed by DWT. The higher frequency components are used for classification. The supervised Support Vector Machine (SVM) classifier is used for classification. Signal classification based on generalized discriminant analysis with kernels is described in [6]. From the generated input signals, features are 
extracted by spectral and statistical methods. SVM classifier is used to classify modulated signals.

A modulation classification scheme in cognitive radios is described in [7]. The amplitude and phase features are extracted by pseudo wigner-ville time frequency distribution. Then, classification is made by decision tree classifier. Neural network based cognitive radio environment is described in [8]. At first, features are extracted from the input generated signals using cyclo-stationary approach, matched filter approach and energy detection approach. The classification is made by artificial neural network.

Blind signal classification is presented in [9] under a cognitive radio networks. Features are extracted by energy time break down kernels. The classification is made by modulation type classifiers. An approach AMC based on temporal waveform features is described in [10]. Initially, the input generated signals are extracted by using temporal features. The classification is made by hierarchical classifier based on multivariate classification. The classification of digital and analog signals using SVM is described in [11]. At first, spectral features are extracted and normalization is employed to normalize the features. The classification is made by SVM classifier.

In this study, an AMC system for SDR using DWT, ICA and PCNN is presented. The organization of paper is as follows: The methods and materials for AMC system such as DWT, ICA and PCNN are discussed in section 2 . The obtained results and discussion of AMC system are described in section 3. Finally, the conclusion of AMC system is given in section 4.

\section{METHODS AND MATERIALS}

The work flow of the AMC system is shown in Fig. 1 which uses three different modulation techniques; QAM, PSK and DPSK to modulate the input signal. Then, AWGN is added to the generated signal as transmission channel before decomposing the modulated signal by DWT. ICA is used for dimensionality reduction of the lower frequency components produced by DWT. The classification of signals is made by PCNN.

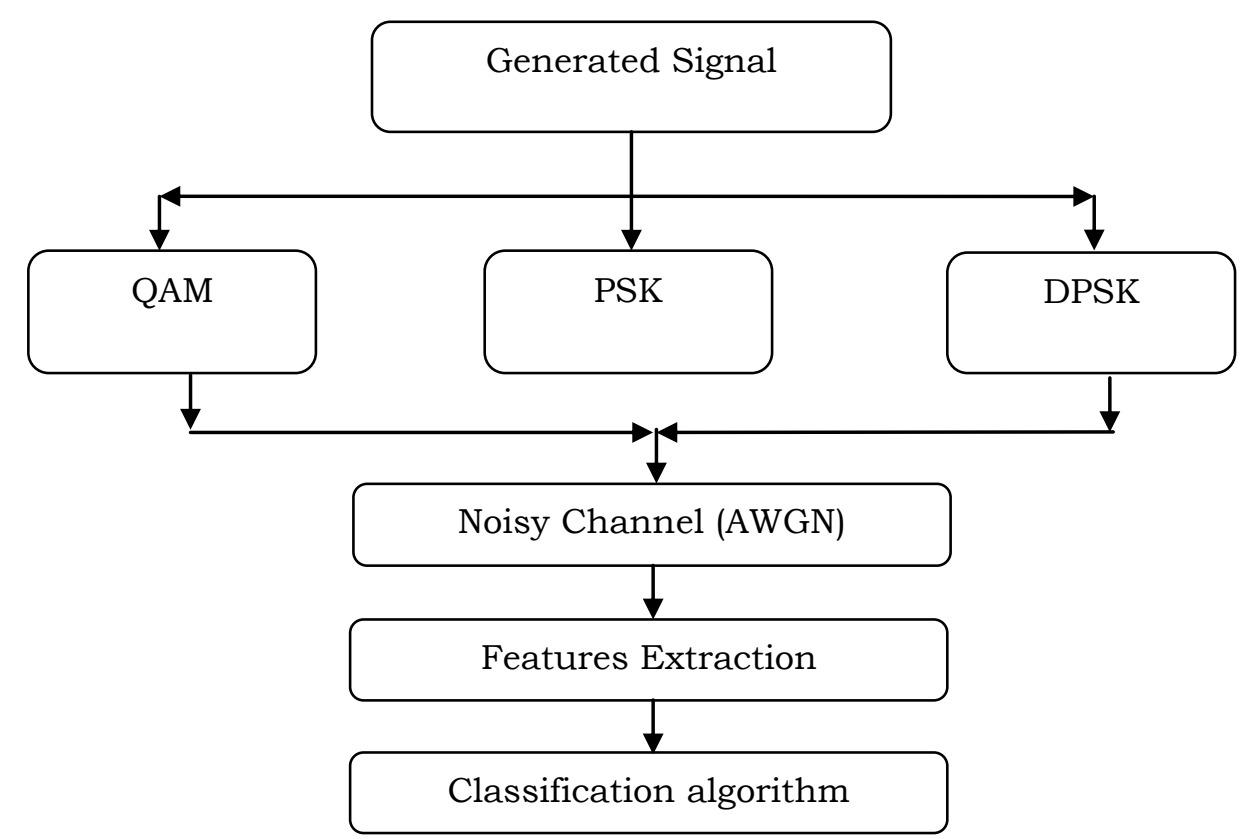


Fig. 1 Workflow of AMC

\section{A. Feature extraction of AMC system}

AWGN is used as a transmission channel model and it is impairment to the communication which is an addition of white noise or wideband with a Gaussian distribution of amplitude and constant spectral density. The channel representation of AWGN is given by the series of outputs as $G_{m}$ at the discrete time of event index $m . G_{m}$ is the sum of input $H_{m}$ and noise, $I_{m}$ is identically distributed and independent drawn between the noise. The $I_{m}$ is assumed to be connected with the $G_{m}$. Then, AWGN is given by,

$$
\frac{1}{l} \sum_{m=1}^{l} g_{m}^{2} \leq V
$$

where $V$ represents the power of the channel. In this study, AWGN noise is added to teh modulated signals of QAM, PSK and DPSK with $0 \mathrm{~dB}, 1 \mathrm{~dB}, 5 \mathrm{~dB}$ and $10 \mathrm{~dB}$ noise densities.

The discretely sampled wavelet transform is known as DWT in functional and numerical analysis. It has a main advantage with a temporal resolution in Fourier transform and both the time and frequency information are captured by DWT. It decomposes the signal into different sub-bands. The DWT is defined by,

$$
\delta(t)=\sum_{k=-\infty}^{\infty}(-1)^{k} L_{N-1-k} \delta(2 t-k)
$$

where $N$ is an even integer to decompose the set of wavelets from the signals. DWT is also used in video hiding technique [12] and watermarking [13]. In this study, the DWT is applied for the decomposition of modulated signal passed through AWGN channel. It produces the lower and higher frequency sub-bands. The lower frequency components are used for dimensionality reduction.

\section{B. ICA based dimensionality reduction}

ICA separates the multivariate signals into additive subcomponents. It is made by the non-Gaussian signals which are subcomponents and they are independent to each other. ICA is specially used in the blind source separation. It has a large database of samples with multivariate data model. It has some data variables with the linear mixtures of unknown latent variables in the unknown system. The latent variables are mutually independent with non-Gaussian signals known as observed data with independent components.

The independent components are also known as factors or sources. ICA is also used in face recognition [14] and image compression [15]. In this study, the ICA is applied only for lower frequency sub-bands which are obtained by DWT. It reduces the dimension for redundant features. Then the reduced features are stored in database for classification. Figure 2 shows the feature extraction stage for AMC system.

\section{PCNN classification}

PCNN performs two-dimensional neural network. It consists of large number of neurons. Each neuron in the PCNN communicates with each data in the input signal and receives information for an external stimulus. Each neuron is connected with their neighboring neurons for receiving the local stimulus from the 
neurons. In the conventional signal or image processing, it has a large number of merits which includes; variations in input patterns for bridging minor intensity and geometric variations, noise robustness and so on. The architecture of PCCN is shown in Fig. 3.

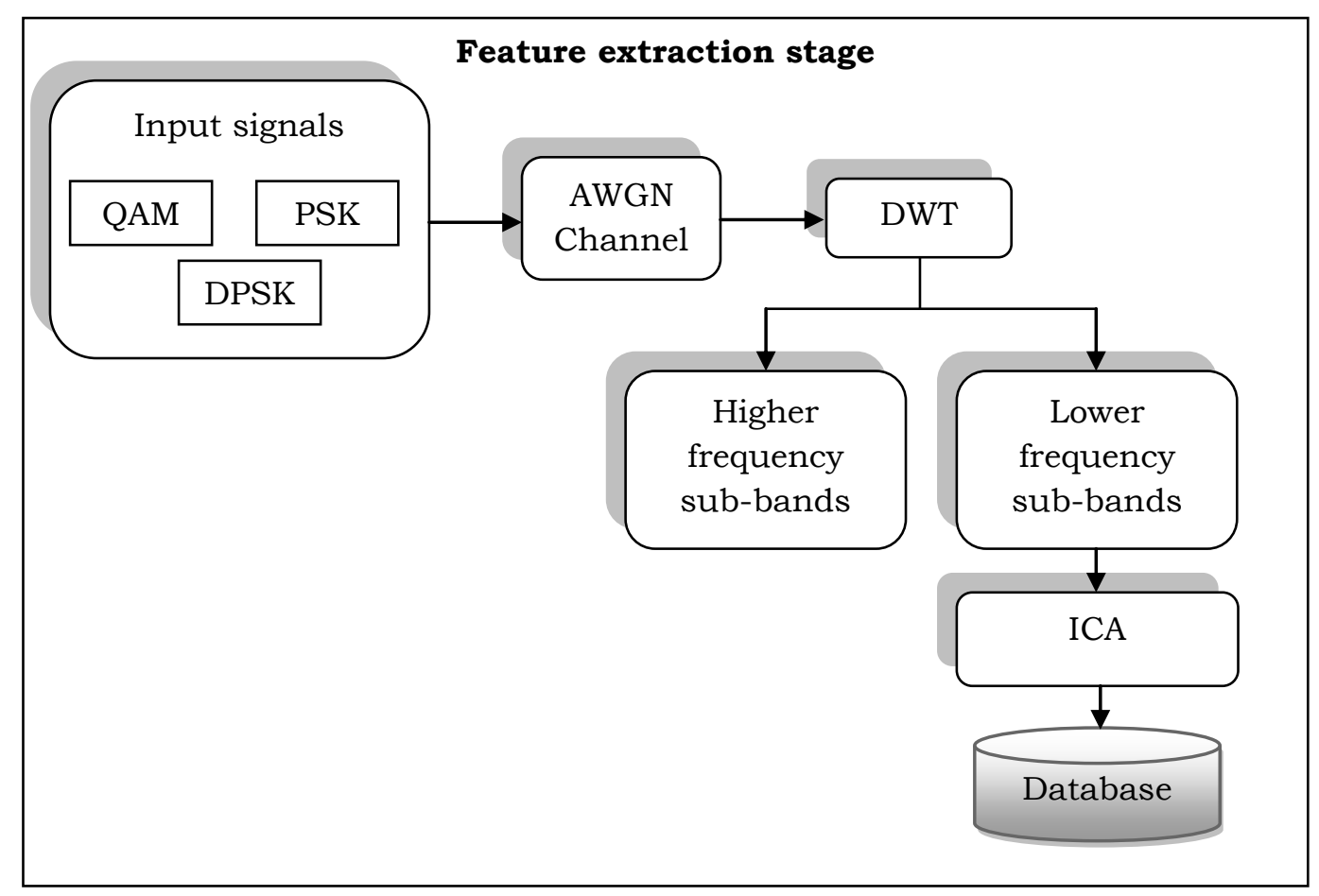

Fig. 2 Feature extraction stage of AMC

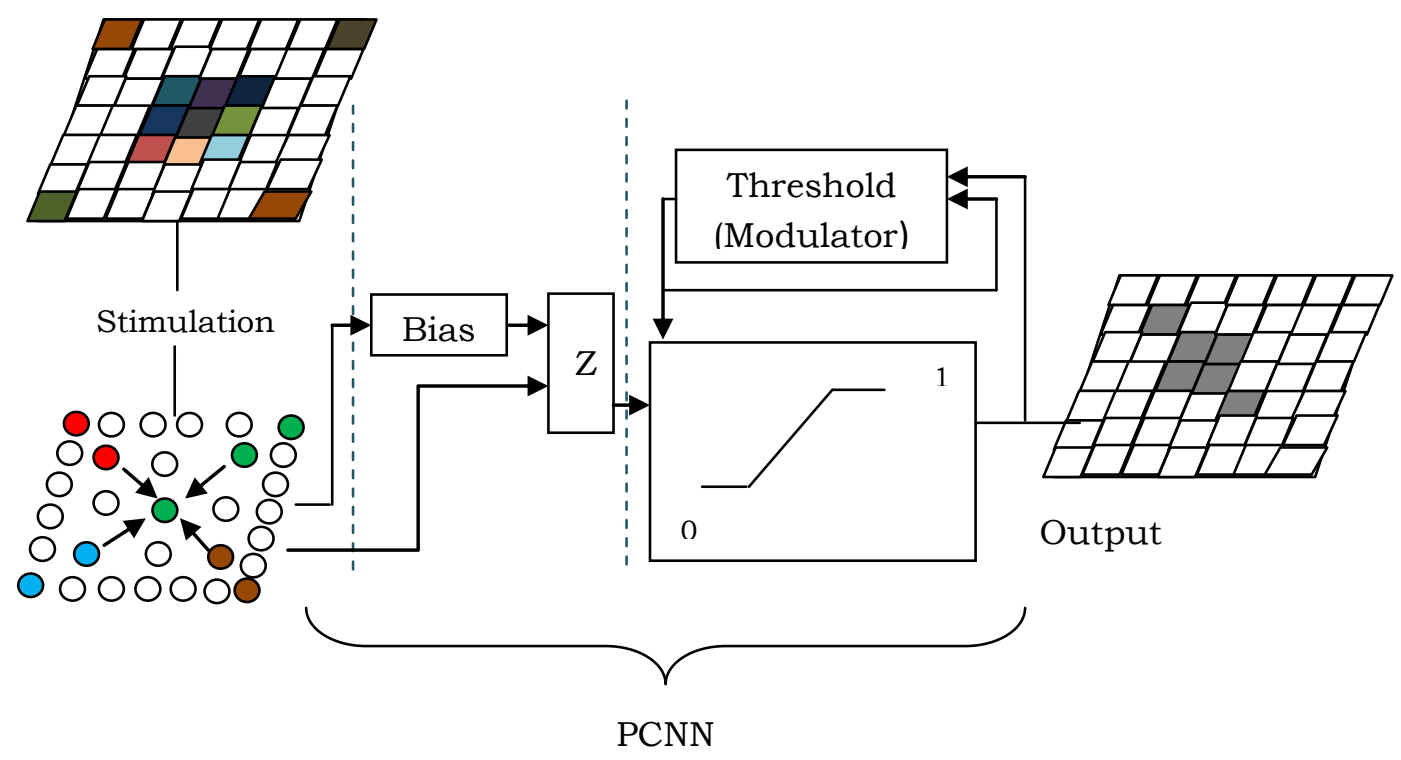

Fig. 3 Architecture of PCNN 
PCNN is also used in multi-object segmentation [16] and region growing [17]. In this study PCNN is used for the classification of the given signals into three digital modulation schemes; QAM, PSK and DPSK. The extracted features are already stored in the feature database in the feature extraction stage. PCNN performs the classification of signals automatically. Figure 4 shows the classification stage of AMC system.

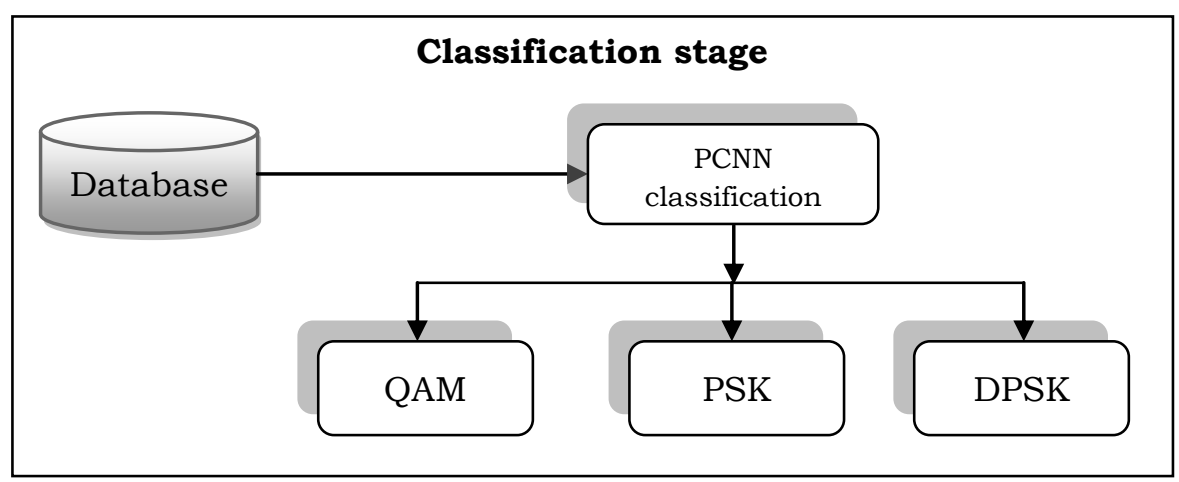

Fig. 4 Classification stage of AMC

\section{RESULTS AND DISCUSSION}

The performance of AMC system is analyzed by using randomly generated 600 signals ( 300 for training and 300 for testing). All the signals are modulated by three different modulation schemes; QAM, PSK and DPSK. These modulated signals are transmitted through AWGN channel with different noise densities such as $0 \mathrm{~dB}, 1 \mathrm{~dB}, 5 \mathrm{~dB}$ and $10 \mathrm{~dB}$. By using the lower frequency components of DWT and ICA the features are extracted with low dimension. Then, the classification is made by PCNN classifier. Table 1 to Table 4 shows the confusion matrix obtained by AMC system.

TABLE 1 Confusion matrix of AMC system at $O \mathrm{~dB}$

\begin{tabular}{|c|c|c|c|}
\hline Type of Modulation & QAM & PSK & DPSK \\
\hline QAM & 52 & 7 & 15 \\
\hline PSK & 40 & 43 & 30 \\
\hline DPSK & 8 & 50 & 55 \\
\hline Accuracy (\%) & \multicolumn{3}{|c|}{$\mathbf{5 0}$} \\
\hline
\end{tabular}

TABLE 2 Confusion matrix of AMC system at $1 \mathrm{~dB}$

\begin{tabular}{|c|c|c|c|}
\hline Type of Modulation & QAM & PSK & DPSK \\
\hline QAM & 85 & 2 & 4 \\
\hline PSK & 12 & 78 & 16 \\
\hline DPSK & 3 & 20 & 80 \\
\hline Accuracy (\%) & \multicolumn{3}{|c|}{$\mathbf{8 1}$} \\
\hline
\end{tabular}


TABLE 3 Confusion matrix of AMC system at $5 \mathrm{~dB}$

\begin{tabular}{|c|c|c|c|}
\hline Type of Modulation & QAM & PSK & DPSK \\
\hline QAM & 95 & 0 & 2 \\
\hline PSK & 0 & 96 & 3 \\
\hline DPSK & 5 & 4 & 95 \\
\hline Accuracy (\%) & \multicolumn{3}{|c|}{$\mathbf{9 5 . 3}$} \\
\hline
\end{tabular}

TABLE 4 Confusion matrix of AMC system at $10 \mathrm{~dB}$

\begin{tabular}{|c|c|c|c|}
\hline Type of Modulation & QAM & PSK & DPSK \\
\hline QAM & 98 & 0 & 0 \\
\hline PSK & 0 & 97 & 3 \\
\hline DPSK & 2 & 3 & 97 \\
\hline Accuracy (\%) & \multicolumn{3}{|c|}{$\mathbf{9 7 . 3}$} \\
\hline
\end{tabular}

From the tables, it is clearly observed that when increasing the noise levels, the performance of the system is reduced. The worst performance occurs at OdB with only $50 \%$ accuracy as the modulated signals contain noises only. When the modulated signals affected by $10 \mathrm{~dB}$ noise, the classification accuracy of the system is $97.3 \%$. Also, it is noted that among the three modulation schemes, QAM signals are more classified than others irrespective of noise densities added the modulated signal.

\section{CONCLUSION}

The AMC system presented in this paper uses DWT, ICA and PCNN techniques for the classification of modulation schemes in the modulated signals. The AWGN channel is used as a transmission channel. DWT is used for the decomposition of signals at $1^{\text {st }}$ level. The feature dimension of lower frequency component of DWT is reduced by ICA. The reduced features are stored in the database to train the PCNN classifier. The different noise intensities; OdB, $1 \mathrm{~dB}$, $5 \mathrm{~dB}$ and $10 \mathrm{~dB}$ are used to contaminate signal and they are used to validate the PCNN classifier. The system produces the better classification accuracy of $97.3 \%$ at $10 \mathrm{~dB}$ noise intensity by PCNN and also the performance of the system degrades when noise is added more than $10 \mathrm{~dB}$.

\section{REFERENCES}

[1]. S.E. El Khamy, A. El Helw, and A. Mahdy, "C25. Digital signal classification by compressed cyclostationary features", National Radio Science Conference, 2012, pp. 363-369.

[2]. F. Benedetto, A. Tedeschi, and G. Giunta, "Automatic blind modulation recognition of analog and digital signals in cognitive radios", Vehicular Technology Conference, 2016, pp. 1-5.

[3]. M. Zamanian, A.A. Tadaion, and M.T. Sadeghi, "Modulation classification of linearly modulated signals in a cognitive radio network using 
constellation shape", International Workshop on Systems, Signal Processing and their Applications, 2011, pp. 13-16.

[4]. B. Ramkumar, T. Bose, and M.S. Radenkovic, "Combined blind equalization and automatic modulation classification for cognitive radios", Digital Signal Processing Workshop and IEEE Signal Processing Education Workshop, 2009, pp. 172-177.

[5]. R. Kannan, and S. Ravi, "Digital signals classification in cognitive radio based on discrete wavelet transform", International Conference on Control Engineering and Communication Technology, 2012, pp. 870-873.

[6]. W. Guibene, and D. Slock, "Signal classification in heterogeneous OFDMbased cognitive radio systems". ICT, 2013, pp. 1-5.

[7]. X. Zhu, and T. Fujii, "Modulation classification in cognitive radios for satellite and terrestrial systems", International Conference on Communication Workshop, 2015, pp. 1612-1616.

[8]. J.J. Popoola, and R. van Olst, "Application of neural network for sensing primary radio signals in a cognitive radio environment", IEEE Africon'11, 2011, pp. 1-6.

[9]. E. Rebeiz, F.L. Yuan, P. Urriza, D. Marković, and D. Cabric, "Energyefficient processor for blind signal classification in cognitive radio networks", IEEE Transactions on Circuits and Systems I: Regular Papers, Vol. 61, No. 2, 2013, pp. 587-599.

[10]. Z. Ye, G. Memik, and J. Grosspietsch, "Digital modulation classification using temporal waveform features for cognitive radios", International Symposium on Personal, Indoor and Mobile Radio Communications, 2007, pp. $1-5$.

[11]. M. Petrova, P. Mähönen, and A. Osuna, "Multi-class classification of analog and digital signals in cognitive radios using support vector machines" International Symposium on Wireless Communication Systems, 2010, pp. 986-990.

[12]. G. Sowmya, K. Meghana, B. Subbarayudu, R.C.A. Naidu, and G. Keerthi, "The video hiding technique based on DWT and genetic algorithm with boolean functions", International Conference on Next Generation Computing Technologies, 2016, pp. 843-849.

[13]. R. Choudhary, and G. Parmar, "A robust image watermarking technique using 2-level discrete wavelet transform (DWT)", International Conference on Communication Control and Intelligent Systems, 2016, pp. 120-124.

[14]. X. Zhang, and X. Ren, "Two dimensional principal component analysis based independent component analysis for face recognition", International Conference on Multimedia Technology, 2011, pp. 934-936.

[15]. V. Singh, S.K. Yadav, N.K. Singh, and P.K. Kalra, "Color image compression using block-based independent component analysis" International Symposium on Image and Signal Processing and Analysis, 2007, pp. 288-292.

[16]. L. Xiaofang, C. Dansong, T. Xianglong, and L. Jiafeng, "Multi-object segmentation based on pulse coupled neural network", Cybernetics and Intelligent Systems, 2008, pp. 744-748.

[17]. N. Chen, Z.B. Qian, S.X. Zhao, and J.S. Fan, "Region Growing Based on Pulse-Coupled Neural Network", International Conference on Machine Learning and Cybernetics, Vol. 5, 2007, pp. 2832-2836. 